Open Access

\title{
A cross-sectional assessment of primary healthcare facilities for provision of antenatal care: calling for improvements in Basic Health Units in Punjab, Pakistan
}

\author{
Muhammad Ashraf Majrooh ${ }^{1 *}$, Seema Hasnain², Javaid Akram² $^{2}$ and Arif Siddiqui ${ }^{2}$
}

\begin{abstract}
Background: There are two types of barriers to the utilisation of maternal health and antenatal care (ANC) services, including the supply-side barriers operating at the health facility level and demand-side, affecting the utilisation ANC services by pregnant women. The purpose of the study was to assess the essential resources required for the provision of ANC services in primary healthcare facilities in Punjab, Pakistan.

Methods: A cross-sectional facility assessment was conducted in primary healthcare facilities across Punjab. A multi-stage sampling was used to randomly select nine districts from three stratifications and 19 primary healthcare facilities in the public sector (17 Basic Health Units (BHUs) and two Rural Health Centres (RHCs)) from each district. A total of 171 health facilities were included. Data on infrastructure and availability of equipment, essential supplies, medicines, treatment protocols, and infection control items was collected through pre-tested, semi-structured questionnaires. Univariate analysis was carried out to describe the frequency and percentages of facilities across three ratings (good, average, and poor) by type of facility.

Results: Overall, $28 \%$ of facilities had poor infrastructure and the availability of equipment was poor in $16 \%$ of the health facilities. Essential supply items, such as urine strips for albumin, blood sugar testing strips, and haemoglobin reagents, were particularly poorly stocked. However, infrastructure and the availability of equipment and supplies were generally better in RHCs compared to BHUs.

Conclusion: Health facilities lacked the resources required to provide quality ANC services, particularly in terms of infrastructure, equipment, supply items, and medicines. The availability of these resources needs to be urgently addressed.

Keywords: Antenatal care, Basic health units, Cross-sectional, Infrastructure, Primary healthcare facilities, Rural health centres
\end{abstract}

\section{Background}

Improving maternal health is one of the eight Millennium Development Goals, with two specific targets focusing on the reduction of the maternal mortality ratio and achieving universal access to reproductive health, including antenatal care (ANC). The most recent maternal mortality ratio for Pakistan was 276 deaths per 100,000 live births

\footnotetext{
* Correspondence: majrooh58@yahoo.com

${ }^{1}$ Professor \& HOD Community Medicine, Services Institute of Medical Sciences (SIMS) Lahore, 34-B, Jatala House, Atchison Housing Society Raiwind Road, Lahore, Pakistan

Full list of author information is available at the end of the article
}

[1]. Further, the proportion of pregnant women who have at least one ANC visit during their pregnancy is $76 \%$, with the proportion of pregnant women having at least four visits decreasing to $37 \%$ [2]. ANC coverage (for one ANC visit) in the Punjab province of Pakistan was estimated at $53 \%$ for 2 years in the 2007-2008 MICS survey [3]. Similarly to the rest of the country, inequity exists between rural and urban areas: coverage is $50 \%$ in rural areas in comparison to $71 \%$ in urban areas.

ANC, a core component of maternal and newborn health services, provides opportunities to reach pregnant women with a number of high-efficacy 
interventions. WHO recommends a minimum of four ANC visits [4] and has specific guidelines on the content of ANC, which should include blood pressure measurement, urine testing for bacteriuria and proteinuria, blood testing to detect syphilis and severe anaemia, and weight/height measurements [5].

The utilisation of maternal health services is a complex phenomenon and is influenced by several factors operating on both the demand and supply sides. Previous studies have provided a multi-level framework to explain factors determining the utilisation of maternal health services [6] and have identified three levels of barriers: the community and household level, the health service delivery level, and the health sector policy and strategic management level [7]. Supply-side barriers operate at the service delivery level and are characteristics of the health system that hinder service uptake [8]; shortages of supplies, drugs, and basic equipment are among these factors. The existing literature mostly identifies the numerous demand-side factors as important barriers to healthcare utilisation in developing countries, but few have addressed supply-side barriers [9].

As reliable information about the availability of drugs, medical equipment, and infrastructure is extremely important, a health facility assessment of firstlevel care facilities was conducted in nine districts of Punjab province, Pakistan. Six areas were assessed: (1) the condition of buildings of primary healthcare (PHC) facilities ("infrastructure"), (2) the availability and functionality of "equipment" (3), the availability of essential "supplies" and (4) "medicines" for provision of ANC services, (5) the availability of "guidelines", and , the availability of "infection control items". We hope that results from this study will assist policymakers to make informed decisions about resource allocations to improve ANC services.

\section{Methodology}

A cross-sectional health facility assessment was conducted between October 2010 and February 2011.

\section{Sampling}

A multi-stage sampling procedure was adopted to select the districts and clusters of health facilities from each district. Details of the sampling methods are presented elsewhere [10]. Briefly, all of the districts in Punjab province were ranked from 1 to 36 on the basis of a composite indicator of sociodemographic and economic development and grouped into three strata: high, medium, and low. A total of nine districts were then selected: districts Gujranwala, Rawalpindi, and Toba Tek Singh from the high stratum; Sargodha, Multan, and Sahiwal from the medium stratum; and Bahawalnagar, Vehari, and Kasur from the low stratum. From each of these districts, a cluster of 19 health facilities (17 Basic Health Units (BHUs) and 2 Rural Health Centres (RHCs)) were randomly selected, making a total of 171 BHUs and RHCs. Health facilities where no health provider was available for interviews on the first visit were replaced $(n=17)$. RHCs are relatively larger $\mathrm{PHC}$ facilities serving a rural population of $10,000-50,000$, while BHUs are relatively smaller health facilities providing $\mathrm{PHC}$ services to a rural population of 5,000-10,000. One BHU is available up to each 'Union Council' level. Union Councils are electoral units in the Punjab consisting of clusters of 2-3 villages or a single large town/village depending upon the size of the population. A uniform package of health services covering the essential package of health services for PHC in Punjab and infrastructure, physical, and human resource requirements are defined for each level of care. Medical Officers manage RHCs/BHUs and ANC services are supposed to be provided by Lady Health Visitors in these facilities [11].

\section{Data collection}

Data relating to the six areas was collected through an objectively developed semi-structured questionnaire, which was pretested in a non-sampled district, Nankana Sahib. The questionnaire was administered through observation, interviews, and examination of facility records by enumerators. For "infrastructure", data were collected on essential amenities in the health facilities (such as phone, electricity, generator, water-supply, toilet facilities, waiting area, privacy for examination, cleanliness, and general condition of the buildings such as boundary wall, gate, signboards, and the approach road). Essential ANC equipment was assessed, including blood pressure apparatus and haemoglobin meters. Availability of essential medicines for pregnant women, such as iron, folic acid, multivitamin, calcium, antimalarial and antipyretic tablets, and tetanus toxoid vaccines, were included in the assessment.

A total of eight teams undertook data collection, with each team consisting of a team leader/supervisor and two surveyors/interviewers. They were given hands-on training in a 3-day workshop on data collection and management in the field. Field monitoring was carried out by two Regional Coordinators, a Public Health Consultant, and the Principal Investigator. Data validation was carried out by inspecting $10 \%$ of records for accuracy.

\section{Data analysis}

For each item in the six areas assessed, univariate analysis described the frequency and percentage of facilities. The facilities that met more than $80 \%$ of the required items were counted as "good" (or "high"), those meeting between $60 \%$ and $80 \%$ as "average" (or "acceptable"), and those meeting less than $60 \%$ as "poor" (or "low"). These 
Table 1 Facility resources of basic health units (BHUs) and rural health centres (RHCs)

\begin{tabular}{|c|c|c|c|c|c|c|}
\hline & Overall & & RHCs & & BHUs & \\
\hline \multirow[t]{2}{*}{ Number of facilities } & 171 & & 18 & & 153 & \\
\hline & $n$ & $\%$ & $\mathrm{n}$ & $\%$ & $\mathrm{n}$ & $\%$ \\
\hline \multicolumn{7}{|l|}{ Infrastructure items } \\
\hline Good general condition (need for repairs and maintenance) & 99 & 58 & 13 & 72 & 86 & 56 \\
\hline Cleanliness (good) & 68 & 40 & 11 & 61 & 57 & 37 \\
\hline Phone (functional) & 91 & 53 & 16 & 89 & 75 & 49 \\
\hline Electricity (available) & 163 & 95 & 18 & 100 & 145 & 95 \\
\hline Electricity (functional) & 160 & 94 & 18 & 100 & 142 & 93 \\
\hline Generator available & 18 & 11 & 15 & 83 & 3 & 2 \\
\hline Generator (functional) & 14 & 8 & 12 & 67 & 2 & 1 \\
\hline Availability of water supply & 165 & 96 & 18 & 100 & 147 & 96 \\
\hline Availability of toilet & 151 & 88 & 18 & 100 & 133 & 87 \\
\hline Separate toilets for males and females & 35 & 20 & 13 & 72 & 22 & 14 \\
\hline Waiting area for patients & 169 & 99 & 18 & 100 & 151 & 99 \\
\hline Privacy for examination of client/pregnant women & 157 & 92 & 17 & 94 & 140 & 92 \\
\hline \multicolumn{7}{|l|}{$\begin{array}{l}\text { Ranking of facilities by availability of infrastructure } \\
\text { (no items available/total items in list) } \times 100 \text { ) }\end{array}$} \\
\hline Good condition $>80 \%$ & 49 & 29 & 17 & 94 & 32 & 21 \\
\hline Average condition 60-80\% & 74 & 43 & 1 & 6 & 73 & 48 \\
\hline Poor condition <60\% & 48 & 28 & - & - & 48 & 31 \\
\hline \multicolumn{7}{|l|}{ Equipment items } \\
\hline Weighing machine available & 139 & 81 & 14 & 78 & 125 & 82 \\
\hline Weighing machine functional & 130 & 76 & 13 & 72 & 117 & 76 \\
\hline Height meter available & 28 & 16 & 6 & 33 & 22 & 14 \\
\hline Height meter functional & 27 & 16 & 6 & 33 & 21 & 14 \\
\hline Thermometer available & 142 & 83 & 15 & 83 & 127 & 83 \\
\hline Blood pressure apparatus available & 164 & 96 & 18 & 100 & 146 & 95 \\
\hline Blood pressure apparatus functional & 161 & 94 & 18 & 100 & 143 & 93 \\
\hline Stethoscope available & 164 & 96 & 18 & 100 & 146 & 95 \\
\hline Stethoscope functional & 163 & 95 & 18 & 100 & 145 & 95 \\
\hline Fetal stethoscope available & 121 & 71 & 18 & 100 & 103 & 67 \\
\hline Exam couch available & 160 & 94 & 18 & 100 & 142 & 93 \\
\hline Refrigerator available & 151 & 88 & 18 & 100 & 133 & 87 \\
\hline Refrigerator functional & 141 & 82 & 18 & 100 & 123 & 80 \\
\hline Availability of vaccine carrier & 160 & 94 & 18 & 100 & 142 & 93 \\
\hline Vaccine carrier functional & 151 & 88 & 16 & 89 & 135 & 88 \\
\hline Syringe cutter available & 148 & 87 & 15 & 83 & 133 & 87 \\
\hline Syringe cutter functional & 144 & 84 & 14 & 78 & 130 & 85 \\
\hline Haemoglobin meter available & 68 & 40 & 18 & 100 & 50 & 33 \\
\hline Haemoglobin meter functional & 54 & 32 & 18 & 100 & 36 & 24 \\
\hline Microscope available & 68 & 40 & 18 & 100 & 50 & 33 \\
\hline Microscope functional & 41 & 24 & 18 & 100 & 23 & 15 \\
\hline Sterilizer available & 132 & 77 & 17 & 94 & 115 & 75 \\
\hline Sterilizer functional & 97 & 57 & 14 & 78 & 83 & 54 \\
\hline
\end{tabular}


Table 1 Facility resources of basic health units (BHUs) and rural health centres (RHCs) (Continued)

Sharps container available

Ranking of facilities by availability of equipment (no items available/total items in list) $\times 100$ )

\section{Good $>80 \%$}

Average 60-80\%

60

84

Poor $<60 \%$

Supplies items

Antenatal care cards

Maternal health register

Health education material

Disposable syringes available

Haemoglobin reagents available

Benedict solution available

Blood sugar testing strips available

Urine strips for albumin available

Slides for malaria parasites available

Disinfectant available

Latex gloves available

5-mL disposable syringe available

Branula available

Soap available

Ranking of facilities by availability of supplies (no items available/total items in list) $\times 100$ )

Adequate supplies $>80 \%$

Acceptable supplies 60-80\%

Poor supplies $<60 \%$

Drugs list

Tetanus toxoid vaccine

Iron tablets

Folic acid tablets

Antimalarial tablets

Antipyretics

Calcium tablets

Multivitamins

Ranking of facilities by availability of drugs (no items available/total items in list) $\times 100$ )

Adequate drug supply $>80 \%$

Acceptable drug supply 60-80\%

Poor drug supply $<60 \%$

Availability and display of guidelines and protocols

Protocol available

Protocol displayed

Infection control items

Disinfectant available

Latex gloves available

Sharps container available 
Table 1 Facility resources of basic health units (BHUs) and rural health centres (RHCs) (Continued)

\begin{tabular}{|c|c|c|c|c|c|c|}
\hline 5-mL disposable syringe available & 128 & 75 & 16 & 89 & 112 & 73 \\
\hline Branula available & 102 & 60 & 16 & 89 & 86 & 56 \\
\hline Soap available & 139 & 81 & 15 & 83 & 124 & 81 \\
\hline \multicolumn{7}{|c|}{$\begin{array}{l}\text { Ranking of facilities by availability of infection control } \\
\text { (no items available/total items in list) } \times 100 \text { ) }\end{array}$} \\
\hline Good infection control >80\% & 93 & 54 & 15 & 83 & 78 & 51 \\
\hline Acceptable control 60-80\% & 55 & 32 & 3 & 17 & 52 & 34 \\
\hline Poor control <60\% & 23 & 13 & - & - & 23 & 15 \\
\hline
\end{tabular}

three categories were generated for ease of interpretation of findings. All analyses were conducted using SPSS software.

\section{Results}

The overall condition of infrastructure was poor in $31 \%$ of BHUs. Only $56 \%$ were classified as good, requiring no repair, and $40 \%$ were considered clean. A functional generator, separate toilets for men and women, and a phone were available in only $1 \%, 14 \%$, and $49 \%$ of BHUs, respectively. In contrast, the overall condition of infrastructure was good in the majority of the RHCs (94\%; Table 1).

The availability and functionality of the equipment was "low" in $18 \%$ of BHUs. Some of the essential equipment, such as height meters, haemoglobin meters, and microscopes, were available in less than $50 \%$ of BHUs. A fetal stethoscope, the most essential equipment for ANC services, was available only in $67 \%$ of BHUs. RHCs fared better in terms of the availability and functionality of equipment with no RHCs considered "poor" and $83 \%$ considered "good".

The availability of supply items was low in $8 \%$ of BHUs; the availability of some essential supply items, such as urine strips for albumin and Benedict's solution, were particularly low as they were available at less than $5 \%$ of BHUs. Haemoglobin reagent and blood sugar testing strips were available in $16 \%$ and $53 \%$ of BHUs. In contrast, the availability of supplies was high in $94 \%$ of RHCs.

The availability of medicines was low in $17 \%$ of BHUs. Calcium tablets and multivitamins were deficient in $71 \%$ and $41 \%$ of BHUs, respectively. The majority (72\%) of RHCs had high availability with adequate medicine supplies but the remaining $28 \%$ had room for improvement.

Service delivery protocols were available in only $75 \%$ of surveyed facilities (74\% in BHUs and $89 \%$ in RHCs) and similar proportions had them displayed in the facility.

The availability of infection control items was low in $15 \%$ of BHUs while all RHCs had adequate or high availability; indeed, $83 \%$ of RHCs had high availability scoring above $80 \%$. The availability of sharps containers was particularly low (67\% in RHCs and 48\% in BHUs). A steriliser, essential equipment for infection control measures, was found to be functional in 54\% of the BHUs.

\section{Discussion}

\section{Key findings}

The current study focused on facility resources, in particular infrastructure and availability of equipment, supplies, drugs, and service protocols, for the provision of ANC. A large majority (80-95\%) of RHCs were considered "good" in terms of infrastructure or had a high availability of equipment, supplies, and infection control items. The availability of medicines could be improved in RHCs, as only $28 \%$ had "acceptable" availability. BHUs fared poorly in comparison to RHCs in all the six areas. In particular, nearly a third of BHUs had "poor" infrastructure and between $8 \%$ and $20 \%$ had "low" availability of equipment, drugs, and infection control items.

\section{Comparisons to other studies}

The present findings are consistent with those from two national health facility assessments conducted by the Technical Resource Facility in 2012 [12] and by the Pakistan Initiative of Mothers and Newborns in 2005 [13]; however, in some areas, the findings are even more dismal. The deficiencies observed in health facilities in Punjab as reported by the Technical Resource Facility were comparatively less than the values reported herein, with only $7 \%$ of outpatient departments and Lady Health Visitors' rooms requiring extensive repair works. However, only about half of BHUs (237 out of 493) were equipped with more than $75 \%$ of the necessary supplies and $33 \%$ had more than $75 \%$ of the required drugs. With regards to RHCs, 96\% (280 out of 291) had more than $50 \%$ of the supply items and $95 \%$ had at least $75 \%$ of the vaccine items available. Over 95\% of the RHCs in Punjab had outpatient departments that were classified as being in a good condition. The majority of the RHCs (280 out of 291) had more than $50 \%$ of the required supplies, with $95 \%$ of RHCs having more than $75 \%$ of the assessed vaccines. The Pakistan Initiative of Mothers and 
Newborns study also showed that essential drugs were not available in most of the BHUs or Mother and Child Health Centres across the nation, and reported that a foetal stethoscope was available in only $39 \%$ of these facilities [13]. In another health facility assessment conducted by the Free and Fair Election Network Governance Monitors in 2010, the buildings of $8 \%$ of the monitored RHCs in Punjab were in decrepit condition [14], lacking cleanliness and basic hygiene. Further, almost $11 \%$ of the monitored RHCs in Punjab did not have latrines or had latrines without running water.

\section{Policy implications}

The findings of the present study, along with whose of previous studies [12-14], has clearly highlighted the poor status of infrastructure and equipment and the low availability of supplies and medicine in Punjab province health facilities, and in particular BHUs. Based on these findings, we call for urgent increases to resource allocation for infrastructure development and maintenance of rural health facilities. Reforms of existing policies on health facility infrastructure and resource management may be required.

\section{Further research}

Many studies have described severe shortages of essential supplies, medications, and human resources. Further research may be needed to understand how these observed supply-side bottlenecks affect different aspects of service provision [1].

\section{Conclusion}

Gaps have been identified in all input modalities of the ANC delivery system in Pakistan. Resources must be diverted to PHC facilities, in particular BHUs, to strengthen facility infrastructure and the provision of equipment and supplies.

\section{Abbreviations \\ ANC: Antenatal care; BHUs: Basic Health Units; PHC: Primary healthcare; RHCs: Rural health centres.}

\section{Competing interests}

The authors declare that there are no competing financial and non-financial interests with any collaborating organisation or funding agency. The study was conducted in the public interest for the improvement of quality of antenatal care services in Punjab.

\section{Authors' contributions}

MAM conceived the idea of the study, developed the proposal and supervised all of the steps from development of data collection tools to completion of draft of the manuscript and was actively involved in pre-testing, review of tools and monitoring the quality of data collection, entry, and analysis as well as having an active role in preparation of the manuscript. SH assisted in the development of the study proposal, data collection tools, supervision and monitoring of data collection, writing the introduction, the discussion, references and overall preparation and review of the manuscript for publication, SH also played a major role in discussion. JA played a major role in review of manuscript.
AS provided technical assistance in the preparation and review of the manuscript. All authors approved the final version of the manuscript.

\section{Acknowledgements}

The project was funded by the Maternal and Newborn Health Programme of the Research and Advocacy Fund (RAF), a key component of the Department for International Development (DfID) and Australian Agency for International Development (AusAID) as part of their support for maternal, newborn, and child health $(\mathrm{MNCH})$ to achieve Millennium Development Goals 4 and 5 in Pakistan. We are grateful for the cooperation of Dr Muhammad Aslam, Director General Health Punjab, Dr Sabiha Khurshid, Director MNCH programme, and Dr Muhammad Anwar Janjua, Director MIS. We appreciate the efforts of the regional coordinators, Inam-ul-Haq and Fayyaz Ahmed Butt, as well as of the team supervisors and male and female field enumerators who made it possible to complete this work on schedule. Particular gratitude is due to the entire field team. All district health managers (EDOH/DOH, district coordinators for $\mathrm{MNCH}$ programme) provided valuable support by contributing the services of their staff. Special thanks are owed to the communities and local leadership of sampled districts who devoted their time and resources to enable the survey teams to complete their work.

\section{Declaration}

Publication costs for this article were funded by the British Council. This article has been published as part of Health Research Policy and Systems Volume 13 Supplement 1, 2015: Maternal and Newborn Health Research and Advocacy Fund, Pakistan. The full contents of the supplement are available online at http://www.health-policy-systems.com/supplements.

\section{Author details}

${ }^{1}$ Professor \& HOD Community Medicine, Services Institute of Medical Sciences (SIMS) Lahore, 34-B, Jatala House, Atchison Housing Society Raiwind Road, Lahore, Pakistan. ${ }^{2}$ Allama lqbal Medical College, Lahore, Pakistan.

Published: 26 November 2015

\section{References}

1. Demographic and Health Survey 2006-07. Islamabad and Calverton, MA: National Institute of Population Studies and Macro International; 2008. https://dhsprogram.com/pubs/pdf/FR200/FR200.pdf.

2. Pakistan Demographic and Health Survey 2012-13. Islamabad and Calverton, MA: National Institute of Population Studies and ICF International; 2013. https://dhsprogram.com/pubs/pdf/FR290/FR290.pdf.

3. Multiple Indicator Cluster Survery. Punjab 2007-08. Lahore: Bureau of Statistics, Planning and Development Department, Government of the Punjab; 2009.

4. World Health Organization. Standards for maternal and neonatal care. Geneva: WHO, Department of Making Pregnancy Safer; 2006. whqlibdoc.who.int/hq/2007/a91272.pdf.

5. World Health Organization. Integrated management of pregnancy and childbirth. Geneva: WHO; 2003. http://www.who.int/ maternal_child_adolescent/topics/maternal/impac/en/.

6. Jacobs B, Ir P, Bigdeli M, Annear PL, Van Damme W. Addressing access barriers to health services: an analytical framework for selecting appropriate interventions in low-income Asian countries. Health Policy Plan. 2012;27(4):288-300.

7. Hanson K, Ranson MK, Oliveira-Cruz V, Mills A. Expanding access to priority health interventions: a framework for understanding the constraints to scaling-up. J Int Dev. 2003;15(1):1-14.

8. O'Donnell O. Access to health care in developing countries: breaking down demand side barriers. Cad Saude Publica. 2007;23(12):2820-34.

9. Metcalfe R, Adegoke AA. Strategies to increase facility-based skilled birth attendance in South Asia: a literature review. Int Health. 2013;5(2):96-105.

10. Majrooh MA, Hasnain S, Akram J, Siddiqui A, Memon ZA. Coverage and quality of antenatal care provided at primary health care facilities in the 'Punjab' province of 'Pakistan'. PLoS One. 2014;9(11):e113390.

11. Technical Resource Facility. Essential package of health services for primary health care in Punjab 2012. Islamabad: TRF and Government of the Punjab Health Department; 2013.

12. Technical Resource Facility. Health facility assessment - Punjab provincial report. Islamanad: TRF; 2012.

13. Pakistan Initiative for Mothers and Newborns. Health facility assessment. Islamabad: PAIMAN; 2005.

14. Fair and Free Election Network. Wider regional disparities in rural health centres nationwide. Islamabad: FAFEN; 2010 